\title{
Wer vertritt Naturheilkunde und Komplementärmedizin?
}

\author{
Andreas Michalsen \\ Innere Medizin und Integrative Medizin, Kliniken Essen-Mitte, Deutschland
}

Naturheilkunde und Komplementärmedizin haben sich in den letzten Jahrzehnten in allen medizinischen Versorgungsstufen, aber auch in der Hochschulmedizin etabliert. Demoskopische Erhebungen bestätigen Jahr für Jahr das große Interesse vieler Menschen, im Krankheitsfalle mit Naturheilverfahren behandelt zu werden. Die wachsende Präsenz der Naturheilkunde und Komplementärmedizin in der Hochschulforschung, die Implementierung der Naturheilverfahren in der Approbationsordnung durch den Querschnittsbereich 12, zahlreiche Publikationen komplementärmedizinischer Studien in hochrangigen internationalen Journalen und die Etablierung mehrerer in Medline gelisteter Fachzeitschriften mit ansteigenden Impact-Faktoren verweisen auf eine erfreuliche Entwicklung. Auch Forschende KOMPLEMENTÄRMEDIZIN trägt zu dieser Entwicklung bei und hat ihren Impact-Faktor in den letzten Jahren auf aktuell 1,417 gesteigert, womit sie international zu den wichtigsten komplementärmedizinischen Journalen gehört.

In der Frage der öffentlichen, wissenschaftlichen und berufsbezogenen Fach- und Interessenvertretung war hingegen in den letzten Jahren keine Weiterentwicklung zu verzeichnen. Zwar existieren in Deutschland gut etablierte ärztliche Vereinigungen, so unter anderem der Zentralverband der Ärzte für Naturheilverfahren und Regulationsmedizin e.V. (ZAEN) mit 8000 Mitgliedern sowie die Ärztegesellschaft für Erfahrungsheilkunde e.V., die auch die sehr erfolgreiche Medizinische Woche in Baden-Baden veranstaltet. Beiden Ärztegesellschaften ist jedoch gemeinsam, dass nur wenige Kontakte und Schnittstellen mit den komplementärmedizinischen Hochschuleinrichtungen bestehen und der wissenschaftliche Diskurs im Hintergrund steht.

Die in den 1990er Jahren auf Initiative von Prof. Malte Bühring gegründete medizinisch wissenschaftliche Fachgesellschaft «European Society for Complementary and Natural Medicine» (ESCNM) und ihre im Jahr 2000 gegründete Deut- sche Sektion, die «Deutsche Gesellschaft für Naturheilkunde e.V.», konnten bislang kaum Mitglieder und Kooperationen aus dem Bereich der niedergelassenen Ärzte gewinnen. Diese Situation unterscheidet sich damit sehr nachteilig gegenüber den konventionellen medizinischen Fächern, wo in der Regel Hochschulvertreter, klinische Einrichtungen und niedergelassene Fachärzte gemeinsam auftreten und in der Fachgesellschaft organisiert sind. Die entsprechenden Jahreskongresse solcher Gesellschaften, wie z.B. der Deutschen Gesellschaft für Kardiologie oder Onkologie, integrieren wissenschaftliche Erkenntnisse, praktische Anwendung sowie berufsbezogene Interessen und sind damit äußerst erfolgreich und lenken die Entwicklung des eigenen Fachgebietes.

Schließlich sind die konventionellen medizinischen Fachgesellschaften in der «Arbeitsgemeinschaft der wissenschaftlichen Medizinischen Fachgesellschaften» (AWMF) gemeinsam organisiert. Die AWMF befasst sich unter anderem mit Fragen der Approbationsordnung, der Weiterbildungsordnung, der Qualitätssicherung oder der Abrechnungsentwicklungen. Der wachsende Einfluss der AWMF macht nicht zuletzt deutlich, dass die Basis einer modernen medizinischen Fachgesellschaft auch in der wissenschaftlichen Dimension ihres Fachgebietes liegt.

Für die Naturheilkunde und Komplementärmedizin ergibt sich also ein dringender Handlungsbedarf. Vor diesem Hintergrund wurde in den letzten Monaten ein «Relaunch» der Deutschen Gesellschaft für Naturheilkunde (DGN) gestartet. Unter dem Vorsitz von Herrn Prof. Gustav Dobos (Essen) und Frau Prof. Karin Kraft (Rostock), jeweilige Lehrstuhlinhaber im Fachgebiet Naturheilkunde, soll die DGN in Zukunft als eine die Wissenschaft und Praxis integrierende Fachgesellschaft für Naturheilkunde und Komplementärmedizin etabliert werden. Auf dem Gebiet der Naturheilkunde und Komplementärmedizin aktive Wissenschaftler und klinisch/ praktisch tätige Mediziner sind aufgerufen, sich aktiv in der

\begin{tabular}{ll}
\hline KARGER & @ 2007 S. Karger GmbH, Freiburg \\
Fax +497614520714 & Accessible online at: \\
$\begin{array}{l}\text { E-mail Information@Karger.de } \\
\text { www.karger.com }\end{array}$ & www.karger.com/fok \\
&
\end{tabular}


DGN zu beteiligen (Näheres unter www.gesellschaftnaturheilkunde.de).

Kurzfristige Ziele der DGN sind die Aufnahme in die AWMF, die Verbesserung der Forschungsförderung und die Qualitätssicherung der naturheilkundlich ärztlichen Berufausübung. Hierbei kann sich die DGN (und die ESCNM im europäischen Rahmen) auch zwischen rein wissenschaftlichen Organisationen (z.B. International Society for Complementary Medicine Research, ISCMR) und den reinen Berufsverbänden (ZAEN etc.) integrierend positionieren. Die Forschende KomplementärMEDIZIN ist hierfür eine wichtige Publikationsplattform, die sowohl die Vernetzung und den wissenschaftlichen Austausch innerhalb und außerhalb der DGN fördern als auch zur Stärkung und Unterstützung der Position der Fachgesellschaft beitragen kann.

In Zukunft wird die DGN außerdem den Preis «Visionär der
Naturheilkunde» für besondere Verdienste und außergewöhnlichen Einsatz für die Naturheilkunde verleihen. Die erstmalige Preisvergabe «Visionär der Naturheilkunde» der DGN erfolgte in diesem Jahr an Herrn Paul Rothenfußer, den Vorsitzenden der Erich Rothenfußer Stiftung in München.

Aktuell unterstützt die Stiftung in diesem Zusammenhang die Mind/Body Medicine Summer School in Essen sowie den Congress on Complementary Medicine Research in München, den Deutschen Akupunkturkongress in Bad Nauheim, die Summer School für klassische Naturheilkunde in Überlingen und das Zentrum für naturheilkundliche Forschung an der TU München.

Auf eine zukünftige erfolgreiche Verzahnung von Wissenschaft, Praxis und Förderern der Naturheilkunde ist zu hoffen. Wir wünschen der DGN eine wachsende aktive Mitgliedschaft! 